\title{
Influência da disponibilidade de reforçadores com diferentes valores sobre respostas de autocontrole
}

The influence of the availability of different values reinforcers over selfcontrol responses

\section{La influencia de la disponibilidad de refuerzadores con valores diferentes en las respuestas de autocontrol}

\author{
Luiz Antonio Bernardes', Roberto Alves Banaco², Denigés Maurel Régis Neto ${ }^{3}$
}

[1] Pontifícia Universidade Católica de São Paulo e Clínica Particular [2] Pontifícia Universidade Católica de São Paulo e Núcleo Paradigma de Análise do Comportamento [3] Pontifícia Universidade Católica de São Paulo e Núcleo Paradigma de Análise do Comportamento | Título abreviado: Disponibilidade de reforçadores e respostas de autocontrole I Endereço para correspondência: Rua Cel Otávio Meyer, 160, sala 230, Centro. Pouso Alegre/MG. CEP 37.550-0 I Email: luaber@yahoo.com.br

\begin{abstract}
Resumo: Este trabalho apresenta os dados coletados sobre situações de escolha de reforçadores, obtidos com a participação de seis mulheres submetidas a uma atividade sobre a qual incidiu uma contingência propiciadora de supressão condicionada. Elas obteriam pontos que poderiam ser poupados ou trocados por produtos de beleza. Era informado que a disponibilidade dos produtos poderia diminuir a cada sessão e que não seriam repostos. Todas participantes pouparam parte dos pontos para terem acesso a produtos de maior valor no início da coleta. Todas gastaram alguma quantidade de pontos pela primeira vez até a terceira sessão. Nas sessões seguintes, com a escassez dos produtos mais baratos, a maioria delas passou a poupar. Uma das participantes apresentou uma mudança no padrão de economia de pontos, optando por gastá-los com produtos de médio custo que já lhe eram acessíveis, à medida que produtos de custo mais baixo se esgotavam. A disponibilidade dos produtos pareceu ter interferido consideravelmente no gastar/poupar pontos. Os dados sugerem que o valor reforçador dos produtos (preferência) pode ser alterado dependendo de variações na sua disponibilidade, ampliando as considerações no paradigma de autocontrole conforme a definição clássica. Parece aqui que poupar não seria sempre a resposta que produziria o "melhor" reforçador.
\end{abstract}

Palavras-chaves: autocontrole, disponibilidade do reforçador, diferentes valores, valor reforçador, escolha. 
Abstract: Self-control is described as a choice that will lead to major long-term consequences in detriment to smaller and immediate consequences. Also can be interpreted as responses that will bring immediate consequences with high probability of occurrence, instead of delayed greater consequences, but low probability of occurrence. This work presents data of choice of reinforcers by women in a parallel study about a conditioned suppression contingency. Six participants obtained points that could be saved or redeemed for beauty products rated according to its commercial value. Participants were informed that the availability of products could decrease from session to session because it was limited initial availability and the products chosen would not be restored. According to the data, all participants save part of the points obtained at the beginning of the collection. All the participants have spent some amount of their first points until the 3rd session. In the following sessions, with a shortage of cheaper products, the participants exchange their points for more expensive products. The availability of the products seemed to have been a factor that interfered considerably in exchange/save points. The data suggest that the product reinforcer value can be changed depend on variations in their availability.

Keywords: self-control, availability of different values, value choice

Resumen: Este trabajo presenta los datos recogidos sobre situaciones de elección de reforzadores, logrados con la participación de seis mujeres que habían experimentado una actividad sobre la cual centró una contingencia de supresión condicionada. Obtendrían puntos que podrían ser salvados o trocados por productos de belleza. Se informó que la disponibilidad de los productos podría disminuir cada sesión y que no serían substituidos. Todos los participantes han ahorrado algunos de los puntos al principio de la colección. Todos han pasado cierta cantidad de puntos por primera vez hasta el tercer sesión. En las siguientes, con una escasez de productos más baratos, la mayoría de ellos pasó de ahorro. Uno de los participantes mostró un cambio en el patrón de puntos de la economía, optan por pasar con productos de medio precio, como productos de menor costo si se terminan. La disponibilidad de los productos parecía haber interferido considerablemente en los gastar/ahorrar puntos. Los datos sugieren que el valor reforzador de los productos puede cambiar dependiendo de las variaciones en su disponibilidad, las consideraciones en el paradigma de autocontrol como la definición clásica de agrandamiento. Aquí parece que no esté salvando siempre la respuesta que produciría la pestaña de "lo mejor".

Palabras-clave: autocontrol, la disponibilidad de diferentes valores, valor de refuerzo, elegida de valor. 
O autocontrole é um fenômeno definido frequentemente por meio de traços de personalidade - o que diverge da ideia de que um indivíduo é capaz de emitir respostas diferentes, em intensidades diferentes (nas mesmas situações ou não), em fases distintas da vida, dependendo do contexto em que estiver inserido (Hanna \& Ribeiro, 2005).

Segundo Pierce e Cheney (2004):

\begin{abstract}
Uma das coisas interessantes sobre situações de autocontrole é que nossas preferências mudam ao longo do tempo. Isto é, podemos valorizar estudar em vez de festejar uma semana antes da festa, mas valorizamos a festa quando a noite da festa chega. Howard Rachlin $(1970,1974)$ e George Ainslie (1975) sugeriram, independentemente, que essas reversões de preferências podem ser analisadas como mudanças na efetividade do reforçamento à medida que aumenta o atraso. O princípio de Ainslie-Rachlin é que o valor do reforçamento diminui à medida que aumenta $o$ atraso entre fazer uma escolha e obter o reforçador. (p. 258)
\end{abstract}

$\mathrm{Na}$ análise do comportamento, o fenômeno intitulado autocontrole tem sido objeto de vários estudos desde a década de 1960, sendo nomeado de diversas maneiras, como automanipulação, autogerenciamento, manipulação de contingências, autoexperimentação, autorregulação e autogoverno (Kerbauy, 2001).

O autocontrole geralmente é visto como a escolha por um curso de ação que leve a consequências maiores em longo prazo em detrimento de respostas que produzam consequências menores e imediatas (Mischel, Ebbesen \& Zeiss, 1972; Rachlin \& Green, 1972). Além disso, ele tem sido estudado como parte do campo de estudo das escolhas (Hanna \& Ribeiro, 2005). No entanto, em algumas situações pode-se interpretar como autocontrolada a resposta que produza consequências imediatas menores, porém com alta probabilidade de ocorrência (Gonçalves, 2005). Por exemplo, em períodos de inflação elevada, um comportamento "impulsivo" tenderia a ser mais reforçado do que o comportamento de poupar, pois os juros decorrentes deste último não seriam suficientes para adquirir, no futuro, os produtos com preços altos. Em outras palavras, ou consumidor compraria no presente ou não teria como comprar no futuro, apesar de ter poupado dinheiro.

Entre os trabalhos voltados à investigação de diferentes variáveis envolvidas no autocontrole, vale citar as pesquisas de Mischel e Ebbesen (1970) e de Mischel, Ebbesen e Zeiss (1972). Eles manipularam a magnitude e o atraso de reforçadores (um pretzel imediato ou dois pretzel atrasados por 15 minutos), bem como a disponibilidade visual desses reforçadores e algumas atividades distrativas (brinquedos disponíveis) durante o período de espera, a fim de esclarecer quais variáveis poderiam determinar a escolha de crianças por reforçadores maiores e atrasados ou por reforçadores menores e imediatos.

Outro estudo importante é o de Grosh e Neuringer (1981). Eles manipularam variáveis semelhantes às de Mischel e Ebbesen (1970) e de Mischel et al. (1972), mas utilizaram ratos como sujeitos experimentais. Os animais também tinham a oportunidade de escolher reforçadores imediatos e menores ou reforçadores atrasados e maiores. Além disso, num dos estudos deste trabalho, estava disponível um disco em que o animal poderia emitir respostas distrativas durante o período requerido para a liberação do reforçador de maior magnitude e maior atraso. Tanto neste estudo quanto nos estudos de Mischel e Ebbesen (1970) e de Mischel et al. (1972), os resultados indicaram que as atividades realizadas pelos sujeitos experimentais durante o período de espera eram importantes para a aquisição ou não do reforçador de maior magnitude e atraso.

No estudo de Moreira e Abreu-Rodrigues (2008), pombos foram expostos a um esquema encadeado de reforçamento semelhante ao proposto por Rachlin (Rachlin \& Green, 1972). O esquema incluía duas possibilidades de escolha: uma delas para o reforçador imediato e de menor magnitude; outra para o reforçador atrasado e de maior magnitude. Neste estudo era exigido que o animal emitisse uma determinada sequência de respostas para a liberação do reforçador de maior magnitude e atraso. $\mathrm{O}$ estudo de Moreira e Abreu-Rodrigues mostrou que responder durante o período de atraso aumentou a frequência de respostas de autocontrole.

O estudo de Matos e Bernardes (2010), por sua vez, apresenta informações importantes a respeito das pesquisas em autocontrole num programa bra- 
sileiro de pós-graduação em análise experimental do comportamento. Entre as informações, estão as variáveis manipuladas em cada pesquisa. Os autores analisaram oito trabalhos nos quais foram manipuladas as seguintes variáveis: (a) atraso e magnitude do reforço, (b) atividades durante o atraso do reforço, (c) aumento e diminuição gradual do período de atraso do reforço, (d) sinalização temporal durante o período de atraso do reforço, (e) atraso de pontos e atraso da troca de pontos, (f) variáveis que evocariam verbalizações sobre eventos privados durante $o$ atraso do reforço, (g) idade e imitação do comportamento de esperar pelo reforço, (h) densidade do reforço e variabilidade de respostas de pressão à barra.

O acréscimo da variável "probabilidade" de reforçamento torna o estudo do autocontrole mais rico do que o promovido pela conceituação tradicional e mais complexo, enquanto arranjo experimental, daquele que tem sido comumente realizado (Matos \& Bernardes, 2010). Para explorar inicialmente o quanto a disponibilidade variável de reforçadores de diferentes magnitudes influenciaria as respostas de poupar (autocontrole) ou de gastar (impulsividade), o presente trabalho apresenta dados coletados sobre situações de escolha de reforçadores, obtidos numa pesquisa sobre supressão condicionada (Régis Neto et al., 2009).

$\mathrm{Na}$ pesquisa conduzida por Régis Neto et al. (2009), as participantes ganhavam pontos pela execução de uma tarefa no computador (i.e., clicar sobre um círculo colorido que se movimentava erraticamente pela tela do computador). $\mathrm{O}$ acúmulo de pontos dava-se por cinco sessões (uma de aquisição da resposta; quatro de reforçamento do desempenho). Na fase experimental (mais três sessões), mediante um estímulo novo (i.e., cor de fundo da tela do computador), a participante tinha seus pontos diminuídos em metade da quantidade obtida até então, em três momentos da sessão aleatoriamente distribuídos. Com isso, admitia-se que a mudança de cor poderia adquirir função supressiva sobre o responder anteriormente reforçado (i.e., respostas de clicar) via pareamento respondente entre a sua introdução e a perda dos pontos obtidos. Por fim, as participantes foram submetidas a uma sessão de extinção do pareamento respondente.

\section{Método}

\section{Participantes}

Seis mulheres, entre 19 e 39 anos, que trabalhavam numa instituição de ensino superior na cidade de São Paulo. Todas as participantes assinaram o Termo de Consentimento Livre e Esclarecido (TCLE) antes de iniciar a coleta da pesquisa.

\section{Tabela 1: Produtos disponíveis para troca de pontos ao longo do experimento.}

Os produtos foram distribuídos em cinco classes de valores. A cada sessão coletada esse número foi decrescendo, a partir da escolha de cada uma das participantes. Portanto, a cada nova sessão de cada participante, poderiam estar subtraídos desse universo de escolha 6 ou mais produtos que estiveram disponíveis na sessão anterior. As células coloridas indicam a escolha da participante naquela sessão resultando no balanço de quantidades de produtos em cada classe para a sessão seguinte.

\begin{tabular}{|c|c|c|c|c|c|}
\hline Preços & $\begin{array}{l}\text { Classe } 1 \\
(7,5)\end{array}$ & $\begin{array}{l}\text { Classe } 2 \\
(15)\end{array}$ & $\begin{array}{l}\text { Classe } 3 \\
(30)\end{array}$ & $\begin{array}{l}\text { Classe } 4 \\
(45)\end{array}$ & $\begin{array}{l}\text { Classe } 5 \\
(60)\end{array}$ \\
\hline $\begin{array}{l}\text { Quantidade } \\
\text { inicial }\end{array}$ & 9 & 10 & 5 & 20 & 7 \\
\hline $\mathrm{AL}(24 / 06)$ & 8 & 10 & 5 & 20 & 7 \\
\hline $\mathrm{CA}(26 / 06)$ & 7 & 10 & 5 & 20 & 7 \\
\hline CA(29/06) & 7 & 9 & 5 & 20 & 7 \\
\hline NA(29/06) & 6 & 8 & 5 & 20 & 7 \\
\hline LU(30/06) & 5 & 6 & 5 & 20 & 7 \\
\hline $\operatorname{MI}(30 / 06)$ & 5 & 5 & 5 & 20 & 7 \\
\hline $\mathrm{MA}(02 / 07)$ & 5 & 4 & 5 & 20 & 7 \\
\hline $\mathrm{CA}(02 / 07)$ & 5 & 2 & 5 & 20 & 7 \\
\hline $\mathrm{MA}(03 / 07)$ & 5 & 2 & 5 & 19 & 7 \\
\hline MI(03/07) & 5 & 2 & 5 & 18 & 7 \\
\hline $\operatorname{AL}(07 / 07)$ & 5 & 0 & 5 & 18 & 7 \\
\hline CA $(07 / 07)$ & 5 & 0 & 5 & 17 & 7 \\
\hline LU(07/07) & 5 & 0 & 5 & 16 & 7 \\
\hline LU(08/07) & 3 & 0 & 5 & 16 & 7 \\
\hline NA(08/07) & 3 & 0 & 5 & 15 & 7 \\
\hline $\operatorname{AL}(08 / 07)$ & 3 & 0 & 4 & 14 & 7 \\
\hline MA(13/07) & 3 & 0 & 2 & 14 & 7 \\
\hline NA (13/07) & 2 & 0 & 2 & 14 & 7 \\
\hline MI(13/07) & 2 & 0 & 0 & 14 & 7 \\
\hline
\end{tabular}




\section{Material e Ambiente}

Os dados foram coletados no Laboratório de Psicologia Experimental do Curso de Psicologia da Faculdade de Ciências Humanas e da Saúde, da Pontifícia Universidade Católica de São Paulo, por alunos do Programa de Estudos PósGraduados em Psicologia Experimental: Análise do Comportamento.

A sala de coleta era composta por um microcomputador equipado com um software utilizado para a coleta de dados e para administração das variáveis de uma pesquisa de supressão condicionada (Regis Neto et al., 2009), uma mesa, uma cadeira e um display de produtos. A Tabela 1 descreve os produtos disponíveis no início da coleta de dados e os "preços" pelos quais as participantes poderiam obtê-los. Esses preços foram definidos pela equipe feminina da coleta de pesquisa, sendo ratificados por uma das pesquisadoras que trabalhava no setor de marketing da empresa que forneceu o material a ser liberado como reforçador.

\section{Procedimento}

As participantes foram submetidas, individualmente, a um procedimento de supressão condicionada. A cada sessão, elas poderiam obter 7.5 pontos, a serem poupados ou trocados imediatamente por produtos de beleza cuja pontuação foi estabelecida conforme o valor de mercado. No display, havia produtos que "custavam" 7.5 pontos (mínimo obtido durante a tarefa desempenhada numa sessão), 15 pontos, 30 pontos, 45 pontos e 60 pontos - total alcançado economizando-se os pontos em várias sessões.

As participantes foram informadas de que a disponibilidade dos produtos poderia diminuir de sessão para sessão, uma vez que a quantidade de produtos disponíveis era limitada ao que havia no display e os produtos escolhidos por elas não seriam repostos. Assim, o que estava disposto na data da coleta não estaria disposto, necessariamente, na próxima sessão.

Ao final de cada sessão, perguntava-se às participantes se elas gostariam de trocar os pontos obtidos até o momento por algum produto disponível ou se prefeririam acumular os pontos para as sessões seguintes. Caso optassem pela troca, elas deveriam indicar o(s) produto(s) escolhido(s) na- quela ocasião, $o(\mathrm{~s})$ qual(is) era(m) imediatamente entregue(s) a elas pelo experimentador. Se optassem pela manutenção dos pontos, estes eram registrados numa folha individualizada para trocas posteriores.

\section{Resultados e Discussão}

De acordo com os dados apresentados na Tabela 2, todas as participantes pouparam parte dos pontos obtidos para obterem os produtos de maior valor. Também se pode observar que todas as participantes gastaram alguns pontos até a terceira sessão, com produtos de preço baixo ou intermediário, já que para adquirir produtos de valores altos ( 45 e 60 pontos) seria necessário poupar pontos por mais de seis sessões, caso só conseguissem o valor mínimo de pontos da sessão (7.5). Até a sexta sessão, portanto, as participantes só poderiam trocar os pontos obtidos por produtos de até 45 pontos, o que significou consumir os produtos mais baratos, diminuindo assim a possibilidade de escolha desses produtos em sessões posteriores. Assim, as escolhas se deram por produtos de menor valor, de obtenção mais imediata e garantida (i.e., maior probabilidade).

A Tabela 1 apresenta os produtos disponíveis a cada sessão, após as trocas efetuadas por cada participante. À medida que as sessões ocorreram, dada a escassez dos produtos mais baratos, a maioria das participantes acumulou pontos para trocar por produtos mais caros ao final do experimento. Notou-se também uma reversão da preferência por produtos mais baratos e imediatos (e.g., 7,5 pontos) quando o conjunto de produtos de 15 pontos desapareceu das possibilidades de escolha. Antes disso, algumas participantes economizaram pontos em várias sessões. No entanto, a partir da diminuição dos produtos de 15 pontos (na Sessão de 02/07), as escolhas pelos produtos de pontos já acumulados passaram a ser frequentes (produtos de 45 pontos nas Sessões de 03/07 a 08/07; produtos de 30 pontos nas sessões seguintes até o final do experimento).

A Tabela 2 mostra os pontos ganhos, gastos e acumulados por cada uma das participantes ao longo do experimento. As participantes tinham a possibilidade de realizar até nove trocas até o fim do estudo. Por exemplo, a Participante MA esperou até cinco sessões para efetuar uma troca. Já as participantes NA, MI e AL aguardaram quatro sessões 
para fazê-lo, enquanto a participante LU esperou apenas duas sessões para efetuar as trocas de pontos por produtos.

Mesmo com a possibilidade de efetuarem trocas a cada sessão, todas as participantes pouparam parte dos pontos obtidos. Isso se evidencia pelo número de trocas efetuadas: as participantes MA, NA, MI e AL fizeram três trocas ao longo do experimento, enquanto as participantes LU e CA fizeram quatro.

Dessa forma, algumas vezes as participantes gastaram apenas parte do que haviam poupado. Isso sugere que elas tenderam a usufruir tanto dos reforçadores imediatos (i.e., gastaram em produtos de maior probabilidade de obtenção, dado o número de produtos disponíveis) quanto dos reforçadores futuros (i.e., pouparam para tentar obter produtos de probabilidade intermediária). Ao gastarem apenas parte dos pontos acumulados, as participantes compraram produtos que já poderiam ter sido adquiridos em sessões anteriores. Como afirmado anteriormente, a escassez de alguns produtos de valor baixo ou intermediário parece ter mudado o padrão de economia. Comprar posteriormente um produto que já poderia ter sido adquirido precisa ser explicado pela mudança em algum elemento além da quantidade de pontos disponíveis: no caso, a única variável observada foi a diferença na "oferta" de produtos para a troca.
Por terem gasto pontos nas sessões iniciais, algumas participantes não conseguiram acumular pontos suficientes para trocarem pelos produtos de maior valor. Como resultado, tiveram de gastar os pontos restantes em produtos de valor intermediário e com maior probabilidade momentânea de obtenção.

Uma hipótese levantada com o presente estudo (a ser investigada experimentalmente) é de que a disponibilidade dos produtos afetou as respostas de gastar ou de acumular pontos. Essa questão parece ser uma variável diferente das tipicamente consideradas nas definições e arranjos experimentais de autocontrole (Matos \& Bernardes, 2010).

Geralmente, os estudos sobre autocontrole envolvem esquemas de reforçamento concorrentes, com manipulações de variáveis distintas: magnitudes e/ou qualidades dos reforçadores utilizados, distâncias temporais entre a resposta de escolha e a entrega do reforçador, entre outras. No presente estudo, além de um esquema concorrente que permitia às participantes pouparem ou gastarem seus pontos, havia ainda outro componente importante: o fato de que as respostas de escolha emitidas pelas participantes influenciariam a resposta da próxima participante. Quando alguma delas gastava os pontos obtidos, a disponibilidade dos produtos era alterada, conforme apresentado na Tabela 1 . $O$ fato

Tabela 2: Pontos ganhos, gastos e acumulados pelas participantes durante todas as sessões'.

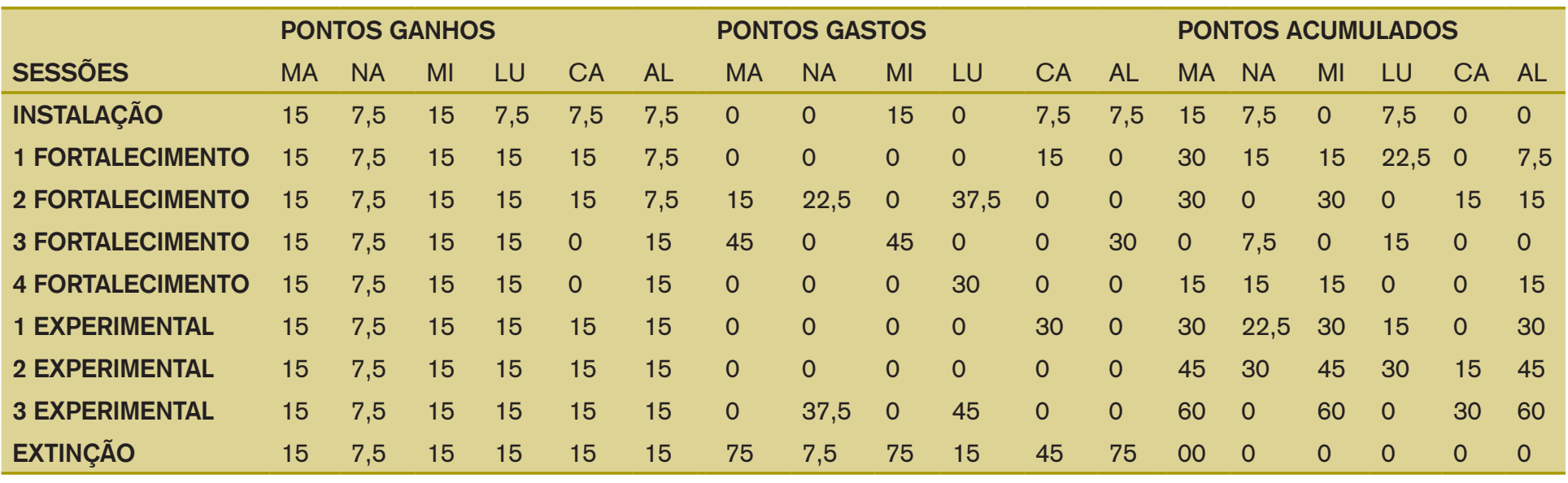

1 CA participou de sete sessões, sendo duas a menos que as demais participantes, por ter atendido o critério de estabilidade delineado pelos experimentadores da pesquisa sobre supressão. 
de a disponibilidade dos reforçadores mudar a cada sessão interferiu, à primeira vista, nas escolhas subsequentes feitas pelas participantes.

Os dados sugerem que (a) a disponibilidade gradativamente menor de produtos de baixo custo e (b) a relativa demora entre as sessões para obter pontos suficientes à troca por produtos de custo maior contribuíram para que as participantes privilegiassem a aquisição de produtos com valor intermediário. Por essa hipótese, pode-se inferir que a demora para a obtenção do reforçador maior vai ao encontro da hipótese de Ainslie-Rachlin, segundo a qual "o valor do reforçamento diminui à medida que aumenta o atraso entre fazer uma escolha e obter um reforçador" (Pierce \& Cheney, 2004, p. 258). Neste caso, poupar era arriscado demais para que a escolha se desse por essa resposta.

Em outras palavras, a resposta de poupar aparentemente não seria a resposta que sempre produziria o "melhor" reforçador, por este poder ser menos provável. Em algumas situações, poupar pode ser indicativo de que esta resposta será reforçada no futuro. Neste caso, porém, como a contingência dependia das respostas emitidas pelas demais participantes, o reforçador escolhido poderia não estar mais disponível para troca. Portanto, existia um risco elevado (uma probabilidade gradativamente menor) para quem escolhia poupar ao invés de gastar na sessão em que produziu os pontos. Dito de outra forma, as respostas de poupar teriam um custo alto e gerariam imprevisibilidade do resultado, enquanto as respostas de gastar envolveriam um custo baixo e maior controle do resultado.

Apesar do caráter introdutório deste trabalho, sugere-se que estudos futuros considerem a importância de (a) realizar um controle sistemático da disponibilidade dos reforçadores, (b) de avaliar em quais produtos os participantes gastam os pontos obtidos e (c) de analisar os efeitos dos intervalos entre as sessões (neste estudo, os intervalos variaram de acordo com a disponibilidade das participantes). Além disso, caberia acrescentar um delineamento que expusesse os participantes a um treino prévio, como linha de base, a fim de observar se os padrões de poupar/gastar se mantêm e também o que os manteriam durante a fase experimental.

Levando em consideração os dados obtidos aqui, o autocontrole não deveria ser visto como um comportamento do tipo "tudo ou nada" (i.e., tem-se ou não se tem), mas um contínuo que poderá ocorrer a depender do tipo de contingência vigente no momento da escolha. Isso porque indivíduos podem ter mais ou menos autocontrole em determinada etapa da vida e sob certas condições (Gonçalves, 2005; Hanna \& Ribeiro, 2005) - fenômeno confirmado neste trabalho em função da disponibilidade do produto, do número de trocas efetuadas e de seu valor reforçador para cada participante.

\section{Referências}

Gonçalves, F. L. (2005). Desvalorização pelo atraso em situações apetitivas e aversivas (Tese de doutorado). Universidade de São Paulo, São Paulo, SP.

Grosh, J. \& Neuringer, A. (1981). Self-control in pigeons under the Mischel paradigma. Journal of the Experimental Analysis of Behavior, 35, 3-21.

Hanna, E. S. \& Ribeiro, M. R. (2005). Autocontrole: Um caso especial de comportamento de escolha. Em J. Abreu-Rodrigues e M. R. Ribeiro (Orgs.). Análise do comportamento: Pesquisa, teoria e aplicação (pp. 175-186). Porto Alegre: Artmed.

Kerbauy R. R. (2001). Acertos e desacertos na pesquisa e aplicação. Em R. Kerbauy (Org.), Sobre comportamento e cognição: Vol. 5. Conceitos, pesquisa e aplicação, a ênfase no ensinar, na emoção e no questionamento clínico (pp.188196). Santo André: ESETec.

Matos, D. \& Bernardes, L. A. (2010). Uma análise das pesquisas de autocontrole do PEXP: Variáveis manipuladas e sua relação com modelos no estudo do autocontrole. Behaviors, 14, 57-68.

Mischel, W. \& Ebbesen, E. (1970). Attention in delay of gratification. Journal of Personality and Social Psychology, 16, 329-337.

Mischel, W., Ebbesen, E. \& Zeiss, A. R. (1972). Cognitive and attentional mechanisms in delay of gratification. Journal of Personality and Social Psychology, 21, 204-218.

Moreira, J. M. \& Abreu-Rodrigues, J. (2008). Atividade durante o atraso: Efeitos sobre a escolha entre autocontrole e impulsividade. Revista Brasileira de Análise do Comportamento, 4, 5370. 
Pierce, W. D. \& Cheney, C. D. (2004). Behavior analysis and learning. London: Lawrence Erlbaum Associates.

Rachlin, H. \& Green, L. (1972). Commitment, choice and self-control. Journal of the Experimental Analysis of Behavior, 17, 15-22.

Regis Neto, D. M. (2009). O efeito de diferentes durações do estímulo condicional na supressão condicionada em humanos. Dissertação de Mestrado defendida na Pontifícia Universidade Católica de São Paulo. Retirado de http:// www.sapientia.pucsp.br/tde_busca/arquivo. php? codArquivo $=8814$

Regis Neto, D. M., Banaco, R. A., Bast, D., Bellodi, A. C., Bernardes, L. A., Bernardo, A. J., Wang, M. A. L. (2009). Supressão condicionada em humanos: Um estudo sobre os efeitos isolados e combinados de estímulos perturbadores. Behaviors, 13, 5-15.

\section{Informações do Artigo}

Histórico do artigo:

Submetido em: 13/05/2013

Primeira decisão editorial: 16/12/2013

Aceito em: 27/03/2014 